\title{
ACCELERATED SPECTRAL REFINEMENT PART II: CLUSTER OF EIGENVALUES
}

\author{
RAFIKUL ALAM ${ }^{1}$, REKHA P. KULKARNI ${ }^{1}$ and BALMOHAN V. LIMAYE ${ }^{1}$
}

(Received 19 December 1995; revised 11 June 1998)

\begin{abstract}
The framework for accelerated spectral refinement for a simple eigenvalue developed in Part I of this paper is employed to treat the general case of a cluster of eigenvalues whose total algebraic multiplicity is finite. Numerical examples concerning the largest and the second largest multiple eigenvalues of an integral operator are given.
\end{abstract}

\section{Introduction}

Spectral refinement for a simple eigenvalue has been widely used in the literature. However, if a simple eigenvalue $\lambda$ is not well-separated from the rest of the spectrum, then for numerical stability it is advisable to consider the cluster of eigenvalues which are close to $\lambda$ and to refine them together. Spectral refinement for a cluster of eigenvalues is considered in [1,2,4-6,13]. In the case of a simple eigenvalue, Dellwo [11] proposed two accelerated refinement schemes which significantly improve the rates of convergence, and in [10], the authors have given a general acceleration procedure. However, so far as we know, accelerated refinement for a multiple eigenvalue, or more generally for a cluster of eigenvalues, has not been reported. In Part I [10] of this paper we introduced a general framework for constructing accelerated refinement schemes for a simple eigenvalue and showed that this approach provides accelerated analogues of many well-known refinement schemes for a simple eigenvalue.

The purpose of the present work is to show that the framework developed in [10] for a simple eigenvalue can be utilized to resolve a multiple eigenvalue or, more generally a cluster $\Lambda$ of nonzero eigenvalues, whose total algebraic multiplicity is finite. This technique is illustrated by considering the fixed-slope Newton scheme. It may be noted that the $q$ th order $(q=2,3, \ldots)$ spectral refinement for a simple

\footnotetext{
'Department of Mathematics, Indian Institute of Technology, Bombay 400 076, India.
}

(C) Australian Mathematical Society 2000, Serial-fee code $0334-2700 / 00$ 
eigenvalue produces a column vector of length $q$ whose first component is expected to approximate a suitable eigenvector. For this reason and also for the purpose of ensuring convergence of the refinement scheme, the first component of the initial eigenvector iterate is normalized appropriately and error estimates are obtained for its iterates. While the same principle can be extended to the case of a cluster of eigenvalues, we propose a normalization of a basis of the approximate spectral subspace rather than a normalization of the first components of the basis elements. As in the case of a simple eigenvalue, the main results are first proved for the case when $\min \{|\lambda|: \lambda \epsilon$ $\Lambda\}>1$. This restriction is then removed by considering a scaling of all the operators involved in the process. The scaling is based on the knowledge of a lower bound for $\min \{|\lambda|: \lambda \in \Lambda\}$. Since each $\lambda$ in the finite set $\Lambda$ is assumed to be nonzero, it seems natural to expect that some (not necessarily sharp) lower bound for $\min \{|\lambda|: \lambda \in \Lambda\}$ is available. Finally, the effectiveness of higher order refinement for a cluster of eigenvalues is illustrated by numerical examples involving an integral operator with multiple eigenvalues.

\section{Refinement for a cluster of eigenvalues}

Let $X$ be a complex Banach space and $B L(X)$ denote the Banach space of all bounded linear operators on $X$ along with the operator norm. Let $\Lambda$ be a cluster of nonzero eigenvalues of $T$ whose total algebraic multiplicity is $m<\infty$ and which is isolated by a curve $\Gamma$ from the rest of $\sigma(T)$ and from 0 . Then $0 \notin \Gamma \cup \operatorname{Int} \Gamma$ and $\sigma(T) \cap$ Int $\Gamma=\Lambda$. As in [9] and [10], for $n=1,2, \ldots$, let $\Delta_{n}=T-T_{n}$ and for a positive integer $q$, consider $\mathbf{X}_{q}=\left\{\left[x_{1}, \ldots, x_{q}\right]^{t}: x_{j} \in X, j=1, \ldots, q\right\}$ and the operators $\mathbf{T}_{q}: \mathbf{X}_{q} \rightarrow \mathbf{X}_{q}, \mathbf{T}_{q, n}: \mathbf{X}_{q} \rightarrow \mathbf{X}_{q}, \mathbf{T}_{q}^{(n)}: \mathbf{X}_{q} \rightarrow \mathbf{X}_{q}$ given by

$$
\begin{aligned}
\mathbf{T}_{q}\left[x_{1}, \ldots, x_{q}\right]^{t} & =\left[T x_{1}, x_{1}, \ldots, x_{q-1}\right]^{t}, \quad\left[x_{1}, \ldots, x_{q}\right]^{t} \in \mathbf{X}_{q}, \\
\mathbf{T}_{q, n}\left[x_{1}, \ldots, x_{q}\right]^{t} & =\left[\sum_{j=0}^{q-1} \Delta_{n}^{j} T_{n} x_{j+1}, x_{1}, \ldots, x_{q-1}\right]^{t}, \quad\left[x_{1}, \ldots, x_{q}\right]^{t} \in \mathbf{X}_{q}, \\
\mathbf{T}_{q}^{(n)}\left[x_{1}, \ldots, x_{q}\right]^{t} & =\left[\sum_{j=0}^{q-2} \Delta_{n}^{j} T_{n} x_{j+1}+\Delta_{n}^{q-1} T x_{q}, x_{1}, \ldots, x_{q-1}\right]^{t},\left[x_{1}, \ldots, x_{q}\right]^{t} \in \mathbf{X}_{q},
\end{aligned}
$$

respectively. The spectral projection $\mathbf{P}_{q}$ associated with $\mathbf{T}_{q}$ and $\Lambda$ is given by

$$
\mathbf{P}_{q}\left[x_{1}, \ldots, x_{q}\right]^{t}=\left[P x_{1}, S_{1} x_{1}, \ldots, S_{q-1} x_{1}\right]^{t}, \quad\left[x_{1}, \ldots, x_{q}\right]^{t} \in \mathbf{X}_{q},
$$

where $P$ is the spectral projection associated with $T$ and $\Lambda$, and

$$
S_{j}=-\frac{1}{2 \pi i} \int_{\Gamma} \frac{(T-z I)^{-1}}{z^{j}} d z, \quad j=1, \ldots, q-1 .
$$


It follows that the map $J_{q}: R(P) \rightarrow R\left(\mathbf{P}_{q}\right)$ given by

$$
J_{q} x=\left[x, S_{1} x, \ldots, S_{q-1} x\right]^{t}, \quad x \in R(P),
$$

is a surjective isomorphism. The adjoint $\mathbf{P}_{q}^{*}$ of $\mathbf{P}_{q}$ is given by

$$
\mathbf{P}_{q}^{*}\left[x_{1}^{*}, \ldots, x_{q}^{*}\right]^{t}=\left[P^{*} x_{1}^{*}+S_{1}^{*} x_{2}^{*}+\cdots+S_{q-1}^{*} x_{q}^{*}, 0, \ldots, 0\right]^{\prime} .
$$

It is easy to see that the map $K_{q}: R\left(P^{*}\right) \rightarrow R\left(\mathbf{P}_{q}^{*}\right)$ given by

$$
K_{q} x^{*}=\left[x^{*}, 0, \ldots, 0\right]^{t}, \quad x^{*} \in R\left(P^{*}\right),
$$

is a surjective isomorphism.

We work under the hypothesis $(\mathrm{H})$ made in [10], that is,

(H): the sequence $\left(\left\|T_{n}\right\|\right)$ is bounded and $\left\|\left(T-T_{n}\right)^{2}\right\| \rightarrow 0$ as $n \rightarrow \infty$.

Then for all large $n$ and all $q=2,3, \ldots, \Gamma$ lies in $\rho\left(\mathbf{T}_{q, n}\right)$ as well as in $\rho\left(\mathbf{T}_{q}^{(n)}\right)$. Moreover, if $r_{\sigma}\left(\Delta_{n}\right)<\min \{|z|: z \in \Gamma\}$, then $\sigma\left(\mathbf{T}_{q}^{(n)}\right) \cap \operatorname{Int} \Gamma=\Lambda$ for all $q=2,3, \ldots$ (see [9, Proposition 3.2(b)] and [10, Proposition 2.2(a)]). Let $\mathbf{P}_{q, n}$ and $\mathbf{P}_{q}^{(n)}$ be the spectral projections associated with $\mathbf{T}_{q, n}$ and $\Lambda_{q, n}=\sigma\left(\mathbf{T}_{q, n}\right) \cap \operatorname{Int} \Gamma$, and $\mathbf{T}_{q}^{(n)}$ and $\Lambda$, respectively.

PROPOSITION 2.1. Assume that $\min \{|\lambda|: \lambda \in \Lambda\}>1$. Then for all large $n$ and all $q=2,3, \ldots$,

$$
\begin{gathered}
\max _{z \in \Gamma}\left\|\left(\mathbf{T}_{q}-z \mathbf{I}_{q}\right)^{-1}\right\|_{\infty} \leq C_{1}, \quad \max _{z \in \Gamma}\left\|\left(\mathbf{T}_{q, n}-z \mathbf{I}_{q}\right)^{-1}\right\|_{\infty} \leq C_{2} \quad \text { and } \\
\max _{z \in \Gamma}\left\|\left(\mathbf{T}_{q}^{(n)}-z \mathbf{I}_{q}\right)^{-1}\right\|_{\infty} \leq C_{3}
\end{gathered}
$$

for some constants $C_{1}, C_{2}$ and $C_{3}$, independent of $q$ and $n$.

Further, for all large $n$ and all $q=2,3, \ldots$,

$$
\operatorname{rank} \mathbf{P}_{q, n}=\operatorname{rank} \mathbf{P}_{q}=\operatorname{rank} \mathbf{P}_{q}^{(n)}=m .
$$

In fact, we have

$$
R\left(\mathbf{P}_{q}^{(n)}\right)=R\left(\mathbf{P}_{q}\right)
$$

PROOF. The existence of constants $C_{1}$ and $C_{2}$ was proved in [9, Theorem 3.3]. Also, by $\left[9\right.$, Theorem 3.5 (a)], $\operatorname{rank} \mathbf{P}_{q, n}=\operatorname{rank} \mathbf{P}_{q}=\operatorname{rank} P=m$ for all large $n$ and all $q=2,3, \ldots$. The existence of a constant $C_{3}$ was proved in [10, Proposition $\left.2.2(\mathrm{~b})\right]$.

Now we show that $R\left(\mathbf{P}_{q}\right) \subset R\left(\mathbf{P}_{q}^{(n)}\right)$. For this purpose, we first prove that $\left(\mathbf{T}_{q}-\right.$ $\left.\mathbf{T}_{q}^{(n)}\right) \mathbf{P}_{q}=\mathbf{0}$. For $\left[x_{1}, \ldots, x_{q}\right]^{t} \in \mathbf{X}_{q}$, we have

$$
\left(\mathbf{T}_{q}-\mathbf{T}_{q}^{(n)}\right) \mathbf{P}_{q}\left[x_{1}, \ldots, x_{q}\right]^{t}=\left[\left(\Delta_{n} P-\sum_{j=1}^{q-2} \Delta_{n}^{j} T_{n} S_{j}-\Delta_{n}^{q-1} T S_{q-1}\right) x_{1}, 0, \ldots, 0\right]^{\prime}
$$


By the identity (2.1) of [10], we see that

$$
\begin{aligned}
\Delta_{n} P & -\sum_{j=1}^{q-2} \Delta_{n}^{j} T_{n} S_{j}-\Delta_{n}^{q-1} T S_{q-1} \\
& =-\frac{1}{2 \pi i} \int_{\Gamma}\left(\Delta_{n}-\sum_{j=1}^{q-2} \frac{\Delta_{n}^{j} T_{n}}{z^{j}}-\frac{\Delta_{n}^{q-1} T}{z^{q-1}}\right) R(z) d z \\
& =-\frac{1}{2 \pi i} \int_{\Gamma}\left(T-z I+\sum_{j=0}^{q-1} \frac{\Delta_{n}^{j}}{z^{j}}(z I-T)\right) R(z) d z \\
& =\frac{1}{2 \pi i} \sum_{j=1}^{q-1} \int_{\Gamma} \frac{\Delta_{n}^{j}}{z^{j}} d z=0,
\end{aligned}
$$

since 0 lies outside $\Gamma$. Thus $\left(\mathbf{T}_{q}-\mathbf{T}_{q}^{(n)}\right) \mathbf{P}_{q}=\mathbf{0}$ for all $q=2,3 \ldots$

But then

$$
\begin{aligned}
\left(\mathbf{P}_{q}-\mathbf{P}_{q}^{(n)}\right) \mathbf{P}_{q} & =-\frac{1}{2 \pi i} \int_{\Gamma}\left[\left(\mathbf{T}_{q}-z \mathbf{I}_{q}\right)^{-1}-\left(\mathbf{T}_{q}^{(n)}-z \mathbf{I}_{q}\right)^{-1}\right] \mathbf{P}_{q} d z \\
& =-\frac{1}{2 \pi i} \int_{\Gamma}\left(\mathbf{T}_{q}^{(n)}-z \mathbf{I}_{q}\right)^{-1}\left(\mathbf{T}_{q}^{(n)}-\mathbf{T}_{q}\right)\left(\mathbf{T}_{q}-z \mathbf{I}_{q}\right)^{-1} \mathbf{P}_{q} d z \\
& =\frac{1}{2 \pi i} \int_{\Gamma}\left(\mathbf{T}_{q}^{(n)}-z \mathbf{I}_{q}\right)^{-1}\left(\mathbf{T}_{q}-\mathbf{T}_{q}^{(n)}\right) \mathbf{P}_{q}\left(\mathbf{T}_{q}-z \mathbf{I}_{q}\right)^{-1} d z \\
& =\mathbf{0}
\end{aligned}
$$

This shows that $R\left(\mathbf{P}_{q}\right) \subset R\left(\mathbf{P}_{q}^{(n)}\right)$. Next, by [10, Proposition 2.2(c)],

$$
\operatorname{rank} \mathbf{P}_{q}^{(n)}=\operatorname{rank} \mathbf{P}_{q, n}=\operatorname{rank} \mathbf{P}_{q}
$$

As both $R\left(\mathbf{P}_{q}\right)$ and $R\left(\mathbf{P}_{q}^{(n)}\right)$ have the same finite dimension and one is contained in the other, it follows that they are equal.

We now introduce some convenient notation; which is consistent with the notations used in [8] and [9].

Consider the linear space $\underline{\mathbf{X}}_{q}=\left\{\left[\mathbf{x}_{1}, \ldots, \mathbf{x}_{m}\right]: \mathbf{x}_{i} \in \mathbf{X}_{q}, i=1, \ldots, m\right\}$ consisting of all $q \times m$ arrays of elements of $X$. For $\underline{\mathbf{x}}=\left[\mathbf{x}_{1}, \ldots, \mathbf{x}_{m}\right]$ in $\underline{\mathbf{X}}_{q}$, let

$$
\|\underline{\mathbf{x}}\|_{\infty}=\max \left\{\left\|\mathbf{x}_{1}\right\|_{\infty}, \ldots,\left\|\mathbf{x}_{m}\right\|_{\infty}\right\} \text {. }
$$

Then $\underline{\mathbf{X}}_{q}$ is a Banach space. When $q=1$, we denote $\underline{\mathbf{X}}_{1}$ simply by $\underline{X}$.

For $\underline{\mathbf{x}}=\left[\mathbf{x}_{1}, \ldots, \mathbf{x}_{m}\right] \in \underline{\mathbf{X}}_{q}$ and an $m \times m$ matrix $A$ with complex entries $a_{i, j}, i, j=$ $1, \ldots, m$, define

$$
\underline{\mathbf{x}} A=\left[\sum_{i=1}^{m} a_{i, 1} \mathbf{x}_{i}, \ldots, \sum_{i=1}^{m} a_{i, m} \mathbf{x}_{i}\right] \in \underline{\mathbf{x}}_{q}
$$


Then

$$
\|\underline{\mathbf{x}} A\|_{\infty} \leq\|\underline{\mathbf{x}}\|_{\infty}\|A\|_{1}
$$

where $\|A\|_{1}$ is the operator 1 -norm of $A$ :

$$
\|A\|_{1}=\max \left\{\sum_{i=1}^{m}\left|a_{i, j}\right|: j=1, \ldots, m\right\} .
$$

Consider the linear space $\underline{\mathbf{X}}_{q}^{*}=\left\{\left[\mathbf{x}_{1}^{*}, \ldots, \mathbf{x}_{m}^{*}\right]: \mathbf{x}_{i}^{*} \in \mathbf{X}_{q}^{*}, i=1, \ldots, m\right\}$ and for $\underline{\mathbf{x}}^{*}=\left[\mathbf{x}_{1}^{*}, \ldots, \mathbf{x}_{m}^{*}\right] \in \underline{\mathbf{X}}_{q}^{*}$, let

$$
\left\|\underline{\mathbf{x}}^{*}\right\|_{1}=\left\|\mathbf{x}_{1}^{*}\right\|_{1}+\cdots+\left\|\mathbf{x}_{m}^{*}\right\|_{1}
$$

Then $\underline{\mathbf{X}}_{q}^{*}$ is a Banach space.

For $\underline{\mathbf{x}}^{*}=\left[\mathbf{x}_{1}^{*}, \ldots, \mathbf{x}_{m}^{*}\right] \in \underline{\mathbf{X}}_{q}^{*}$ and an $m \times m$ matrix $B$ with complex entries $b_{i, j}, i, j=$ $1, \ldots, m$, we have

$$
\left\|\underline{\mathbf{x}}^{*} B\right\|_{1} \leq\left\|\underline{\mathbf{x}}^{*}\right\|_{1}\|B\|_{\infty}
$$

where $\|B\|_{\infty}$ is the operator $\infty$-norm of $B$ :

$$
\|B\|_{\infty}=\max \left\{\sum_{j=1}^{m}\left|b_{i, j}\right|: i=1, \ldots, m\right\} .
$$

We identify $\underline{\mathbf{X}}_{q}^{*}$ with the adjoint space of $\underline{\mathbf{X}}_{q}$.

For $\underline{\mathbf{x}} \in \underline{\mathbf{X}}_{q}$ and $\underline{\mathbf{x}}^{*} \in \underline{\mathbf{X}}_{q}^{*}$, let $\left[\underline{\mathbf{x}}, \underline{\mathbf{x}}^{*}\right]$ denote the $m \times m$ matrix whose $(i, j)$ th entry is $\left\langle\mathbf{x}_{j}, \mathbf{x}_{i}^{*}\right\rangle$. Then

$$
\left\|\left[\underline{\mathbf{x}}, \underline{\mathbf{x}}^{*}\right]\right\|_{1} \leq\|\underline{\mathbf{x}}\|_{\infty}\left\|\underline{\mathbf{x}}^{*}\right\|_{1}
$$

Also, for $m \times m$ matrices $A$ and $B$ with complex entries, we have

$$
\left[\underline{\mathbf{x}} A, \underline{\mathbf{x}}^{*} B\right]=B^{H}\left[\underline{\mathbf{x}}, \underline{\mathbf{x}}^{*}\right] A
$$

where $B^{H}$ denotes the conjugate transpose of the matrix $B$.

For $\mathbf{T} \in B L\left(\mathbf{X}_{q}\right)$, let $\underline{\mathbf{T}}: \underline{\mathbf{X}}_{q} \rightarrow \underline{\mathbf{X}}_{q}$ be defined by

$$
\underline{\mathbf{I}}\left[\mathbf{x}_{1}, \ldots, \mathbf{x}_{m}\right]=\left[\mathbf{T x}_{1}, \ldots, \mathbf{T} \mathbf{x}_{m}\right]
$$

Then $\underline{\mathbf{T}} \in B L\left(\underline{\mathbf{X}}_{q}\right)$ and $\|\underline{\mathbf{T}}\|_{\infty}=\|\mathbf{T}\|_{\infty}$. It is easy to see that

$$
\left[\underline{\mathbf{T x}}, \underline{\mathbf{x}}^{*}\right]=\left[\underline{\mathbf{x}}, \underline{\mathbf{T}}^{*} \underline{\mathbf{x}}^{*}\right], \quad \underline{\mathbf{x}} \in \underline{\mathbf{X}}_{q}, \underline{\mathbf{x}}^{*} \in \underline{\mathbf{X}}_{q}^{*}
$$


Also, if $A$ is an $m \times m$ matrix with complex entries, then

$$
\underline{\mathbf{T}}(\underline{\mathbf{x}} A)=(\underline{\mathbf{T}} \mathbf{x}) A, \quad \underline{\mathbf{x}} \in \underline{\mathbf{x}}_{q} .
$$

Let $\phi_{1}, \ldots, \phi_{m}$ be a basis of the spectral subspace $R(P)$ associated with the operator $T$ and the cluster $\Lambda \subset \sigma(T) \backslash\{0\}$. Let $\phi_{1}^{*}, \ldots, \phi_{m}^{*}$ be the basis of $R\left(P^{*}\right)$ such that

$$
\left\langle\phi_{j}, \phi_{i}^{*}\right\rangle=\delta_{i, j}, \quad i, j=1, \ldots, m .
$$

Consider

$$
\underline{\phi}=\left[\phi_{1}, \ldots, \phi_{m}\right] \in \underline{X} \quad \text { and } \quad \underline{\phi}^{*}=\left[\phi_{1}^{*}, \ldots, \phi_{m}^{*}\right] \in \underline{X}^{*} .
$$

Then $\left[\underline{\phi}, \underline{\phi}^{*}\right]=I_{m}$, where $I_{m}$ is the $m \times m$ identity matrix. Let $\underline{\Phi}_{q}=\underline{J}_{q} \underline{\phi}$ and $\underline{\Phi}_{q}^{*}=\underline{K}_{q} \underline{\phi}^{*}$. Then $\Phi_{q}$ gives an ordered basis of $R\left(\mathbf{P}_{q}\right)$ and $\underline{\Phi}_{q}^{*}$ gives the ordered basis of $R\left(\mathbf{P}_{q}^{*}\right)$ such that

$$
\left[\underline{\Phi}_{q}, \underline{\Phi}_{q}^{*}\right]=\left[\underline{\phi}, \underline{\phi}^{*}\right]=I_{m}
$$

Also, $\left\|\Phi_{q}^{*}\right\|_{1}=\left\|\underline{\phi}^{*}\right\|_{1}$.

By analogy with the accelerated fixed-slope Newton scheme for approximating a nonzero simple eigenvalue $\lambda$ of $T$, we consider such a scheme for approximating the given cluster $\Lambda$ of nonzero eigenvalues of $T$.

By [9, Theorem 3.5(a)], $\operatorname{rank} \mathbf{P}_{q, n}=\operatorname{rank} \mathbf{P}_{q}=m$ for all all large $n$ and all $q=2,3, \ldots$ Let $\left\{\Phi_{q, n, 1}, \ldots, \Phi_{q, n, m}\right\}$ be an ordered basis of the spectral subspace $R\left(\mathbf{P}_{q, n}\right)$ associated with $\mathbf{T}_{q, n}$ and $\Lambda_{q, n}=\sigma\left(\mathbf{T}_{q, n}\right) \cap \operatorname{Int} \Gamma$. There is a unique ordered basis $\left\{\Phi_{q, n, 1}^{*}, \ldots, \Phi_{q, n, m}^{*}\right\}$ of $R\left(\mathbf{P}_{q, n}^{*}\right)$ such that

$$
\left\langle\Phi_{q, n, j}, \Phi_{q, n, i}^{*}\right\rangle=\delta_{i, j}, \quad i, j=1, \ldots, m
$$

Let $\underline{\Phi}_{q, n}=\left[\Phi_{q, n, 1}, \ldots, \Phi_{q, n, m}\right] \in \underline{\mathbf{X}}_{q}$ and $\underline{\Phi}_{q, n}^{*}=\left[\Phi_{q, n, 1}^{*}, \ldots, \Phi_{q, n, m}^{*}\right] \in \underline{\mathbf{X}}_{q}^{*}$. Then

$$
\left[\underline{\Phi}_{q, n}, \underline{\Phi}_{q, n}^{*}\right]=I_{m}
$$

It follows that

$$
\underline{\mathbf{P}}_{q, n} \underline{\mathbf{x}}=\underline{\Phi}_{q, n}\left[\underline{\mathbf{x}}, \underline{\Phi}_{q, n}^{*}\right], \quad \underline{\mathbf{x}} \in \underline{\mathbf{X}}_{q} .
$$

Since $R\left(\mathbf{P}_{q, n}\right)$ is invariant under $\mathbf{T}_{q, n}$, we have

$$
\underline{\mathbf{T}}_{q, n} \Phi_{q, n}=\Phi_{q, n} L_{q, n}
$$

for some $m \times m$ matrix $L_{q, n}$ with complex entries. In fact,

$$
L_{q, n}=\left[\underline{\Phi}_{q, n} L_{q, n}, \underline{\Phi}_{q, n}^{*}\right]=\left[\underline{\mathbf{T}}_{q, n} \underline{\Phi}_{q, n}, \underline{\Phi}_{q, n}^{*}\right]
$$


As the matrix $L_{q, n}$ represents the operator $\left.\mathbf{T}_{q, n}\right|_{R\left(\mathbf{P}_{q, n}\right)}$ with respect to the ordered basis $\Phi_{q, n, 1}, \ldots, \Phi_{q, n, m}$ of $R\left(\mathbf{P}_{q, n}\right)$, the spectral decomposition theorem shows that

$$
\sigma\left(L_{q, n}\right)=\sigma\left(\left.\mathbf{T}_{q, n}\right|_{R\left(\mathbf{P}_{q, n}\right)}\right)=\sigma\left(\mathbf{T}_{q, n}\right) \cap \operatorname{Int} \Gamma=\Lambda_{q, n} .
$$

If $z \in \rho\left(\mathbf{T}_{q, n}\right)$, then it is easy to see that

$$
\begin{aligned}
\left(\underline{\mathbf{T}}_{q, n}-z \underline{\mathbf{I}}_{q}\right)^{-1} \Phi_{q, n} & =\Phi_{q, n}\left(L_{q, n}-z I_{m}\right)^{-1} \quad \text { and } \\
{\left[\left(\underline{\mathbf{T}}_{q, n}-z \underline{\mathbf{I}}_{q}\right)^{-1} \underline{\Phi}_{q, n}, \underline{\Phi}_{q, n}^{*}\right] } & =\left(L_{q, n}-z I_{m}\right)^{-1} .
\end{aligned}
$$

We define $\mathscr{L}_{q, n}: \underline{\mathbf{X}}_{q} \rightarrow \underline{\mathbf{X}}_{q}$ by

$$
\mathscr{L}_{q, n} \underline{\mathrm{x}}=\underline{\mathrm{x}} L_{q, n}, \quad \underline{\mathrm{x}} \in \underline{\mathrm{X}}_{q},
$$

and the block-reduced resolvent $\Sigma_{q, n}: \underline{\mathbf{X}}_{q} \rightarrow \underline{\mathbf{X}}_{q}$ associated with $\mathbf{T}_{q, n}$ and $\Lambda_{q, n}$ by

$$
\Sigma_{q, n} \underline{\mathbf{x}}=-\frac{1}{2 \pi i} \int_{\Gamma}\left(\underline{\mathbf{T}}_{q, n}-z \underline{\mathbf{I}}_{q}\right)^{-1} \underline{\mathbf{x}}\left(L_{q, n}-z I_{m}\right)^{-1} d z, \quad \underline{\mathbf{x}} \in \underline{\mathbf{X}}_{q}
$$

Then by [8, Proposition 1.1], we have

(i) The operators $\underline{\mathbf{T}}_{q, n}, \underline{\mathbf{P}}_{q, n}, \mathscr{L}_{q, n}$ and $\Sigma_{q, n}$ commute;

(ii) $\left(\underline{\mathbf{T}}_{q, n}-\mathscr{L}_{q, n}\right) \Sigma_{q, n}=\underline{\mathbf{I}}_{q}-\underline{\mathbf{P}}_{q, n}$;

(iii) $\Sigma_{q, n} \underline{\mathbf{P}}_{q, n}=\underline{\mathbf{0}}$.

Proceeding exactly the same way as in the case of a simple eigenvalue treated in [10], we obtain the following fixed-slope Newton scheme of order $q$ : for a fixed $n$ large enough and a fixed $q=2,3 \ldots$,

$$
\begin{aligned}
& L_{q, n}^{(0)}:=L_{q, n}, \quad \Phi_{q, n}^{(0)}:=\Phi_{q, n} \text { and for } j=1,2, \ldots, \\
& L_{q, n}^{(j)}:=\left[\mathbf{T}_{q}^{(n)} \Phi_{q, n}^{(j-1)}, \Phi_{q, n}^{*}\right], \\
& \underline{\Phi}_{q, n}^{(j)}:=\Phi_{q, n}^{(j-1)}-\Sigma_{q, n}\left[\underline{\mathbf{T}}_{q}^{(n)} \Phi_{q, n}^{(j-1)}-\underline{\Phi}_{q, n}^{(j-1)} L_{q, n}^{(j)}\right] .
\end{aligned}
$$

Since $\underline{\mathbf{P}}_{q, n} \Sigma_{q, n}=\underline{\mathbf{0}}$ for all $j=1,2, \ldots$, we have

$$
\underline{\mathbf{P}}_{q, n} \underline{\Phi}_{q, n}^{(j)}=\underline{\mathbf{P}}_{q, n} \Phi_{q, n}^{(j-1)}=\cdots=\underline{\mathbf{P}}_{q, n} \underline{\Phi}_{q, n}=\underline{\Phi}_{q, n},
$$

so that

$$
\left[\Phi_{q, n}^{(j)}, \underline{\Phi}_{q, n}^{*}\right]=\left[\Phi_{q, n}^{(j)}, \underline{\mathbf{P}}_{q, n}^{*} \underline{\Phi}_{q, n}^{*}\right]=\left[\underline{\mathbf{P}}_{q, n} \underline{\Phi}_{q, n}^{(j)}, \underline{\Phi}_{q, n}^{*}\right]=\left[\underline{\Phi}_{q, n}, \underline{\Phi}_{q, n}^{*}\right]=I_{m} .
$$

Let $\Phi_{q, n}^{(j)}=\left[\Phi_{q, n, 1}^{(j)}, \ldots, \Phi_{q, n, m}^{(j)}\right]$ and $\phi_{q, n, 1}^{(j)}, \ldots, \phi_{q, n, m}^{(j)}$ denote the first components of $\Phi_{q, n, 1}^{(j)}, \ldots, \Phi_{q, n, m}^{(j)}$, respectively. Then the first row $\phi_{q, n}^{(j)}=\left[\phi_{q, n, 1}^{(j)}, \ldots, \phi_{q, n, m}^{(j)}\right]$ of the $q \times m$ array $\underline{\Phi}_{q, n}^{(j)} \in \underline{\mathbf{X}}_{q}$ is supposed to approximate a basis of the spectral subspace $R(P)$ associated with $T$, and the weighted arithmetic mean of the eigenvalues of the $m \times m$ matrix $L_{q, n}^{(j)}$ is supposed to approximate the weighted arithmetic mean of the eigenvalues of $T$ belonging to $\Lambda$. 


\section{Error estimation}

We first consider the case when $\min \{|\lambda|: \lambda \in \Lambda\}>1$. Then the curve $\Gamma$ isolating $\Lambda$ from the rest of the spectrum of $T$ and from 0 can be so chosen that $\min \{|z|: z \in \Gamma\}>1$.

LEMMA 3.1. Suppose that $\min \{|\lambda|: \lambda \in \Lambda\}>\epsilon \geq 1$. Let $c_{1}=\max _{z \in \Gamma}\left\|(T-z I)^{-1}\right\|$ and consider constants $C_{2}, C_{3}$ ensured in Proposition 2.1 .

(a) Then for all $q=2,3, \ldots$,

$$
\begin{aligned}
\left\|\underline{\Phi}_{q}\right\|_{\infty} & \leq \max \left\{1, \frac{\ell(\Gamma) c_{1}}{2 \pi}\right\}\|\underline{\phi}\|_{\infty}, \\
\left\|\left(\underline{\mathbf{T}}_{q}^{(n)}-\underline{\mathbf{T}}_{q, n}\right) \Phi_{q}\right\|_{\infty} & \leq \frac{c_{1} \ell(\Gamma)\|\underline{\phi}\|_{\infty}}{2 \pi} \frac{\left\|\Delta_{n}^{q} T\right\|}{\epsilon^{q}} \quad \text { for } n=1,2, \ldots, \\
\left\|\mathbf{P}_{q, n}\right\|_{\infty} & \leq \frac{\ell(\Gamma) C_{2}}{2 \pi}, \quad\left\|\underline{\mathbf{P}}_{q, n} \underline{\Phi}_{q}-\Phi_{q}\right\|_{\infty} \leq C \frac{\left\|\Delta_{n}^{q} T\right\|}{\epsilon^{q}} \quad \text { for all large } n,
\end{aligned}
$$

where $C=c_{1} C_{2} C_{3} \max \left\{1, \ell(\Gamma) c_{1} / 2 \pi\right\}\left((\ell(\Gamma) / 2 \pi)\|\phi\|_{\infty}\right)^{2}\left\|\underline{\phi}^{*}\right\|_{1}$ and

$$
\left\|\Sigma_{q, n}\right\|_{\infty} \leq \frac{\ell(\Gamma)}{2 \pi} C_{2}^{2}\left\|\Phi_{q, n}\right\|_{\infty}\left\|\Phi_{q, n}^{*}\right\|_{1} \quad \text { for all large } n .
$$

(b) If $M_{q, n}=\left[\Phi_{q}, \Phi_{q, n}^{*}\right]$ and $N_{q, n}=\left[\Phi_{q, n}, \Phi_{q}^{*}\right]$, then $N_{q, n} M_{q, n} \rightarrow I_{m}$ as $n \rightarrow \infty$, uniformly in $q=2,3, \ldots$

PROOF. We can assume that $\min \{|z|: z \in \Gamma\}>\epsilon \geq 1$.

(a) Since $\underline{\Phi}_{q}=\underline{J}_{q} \underline{\phi}=\left[J_{q} \phi_{1}, \ldots, J_{q} \phi_{m}\right]$, the $(j, i)$ th entry of the $q \times m$ array $\Phi_{q}$ is given by

$$
S_{j-1} \phi_{i}=-\frac{1}{2 \pi i} \int_{\Gamma} \frac{(T-z I)^{-1} \phi_{i}}{z^{j-1}} d z, \quad j=1, \ldots, q, i=1, \ldots, m,
$$

where $\underline{\phi}=\left[\phi_{1}, \ldots, \phi_{m}\right]$ and $S_{0}=P$. Since $\phi_{i} \in R(P), i=1, \ldots, m$ and $|z|>\epsilon \geq 1$ for all $z \in \Gamma$, we see that

$$
\left\|\underline{\Phi}_{q}\right\|_{\infty} \leq \max \left\{1,\left\|S_{1}\right\|, \ldots,\left\|S_{q-1}\right\|\right\}\|\underline{\phi}\|_{\infty} \leq \max \left\{1, \frac{\ell(\Gamma) c_{1}}{2 \pi}\right\}\|\underline{\phi}\|_{\infty} .
$$

Next,

$$
\left(\underline{\mathbf{T}}_{q}^{(n)}-\underline{\mathbf{T}}_{q, n}\right) \Phi_{q}=\left[\left(\mathbf{T}_{q}^{(n)}-\mathbf{T}_{q, n}\right) J_{q} \phi_{1}, \ldots,\left(\mathbf{T}_{q}^{(n)}-\mathbf{T}_{q, n}\right) J_{q} \phi_{m}\right],
$$


where for $i=1, \ldots, m$,

$$
\begin{aligned}
\left(\mathbf{T}_{q}^{(n)}-\mathbf{T}_{q, n}\right) J_{q} \phi_{i}=\left[\Delta_{n}^{q} S_{q-1} \phi_{i}, 0 \ldots, 0\right]^{t}, \\
\left\|\Delta_{n}^{q} S_{q-1} \phi_{i}\right\|=\left\|\frac{\Delta_{n}^{q}}{2 \pi} \int_{\Gamma} \frac{(T-z I)^{-1} \phi_{i}}{z^{q-1}} d z\right\|=\left\|\frac{\Delta_{n}^{q}}{2 \pi} \int_{\Gamma} \frac{\left[T(T-z I)^{-1}-I\right] \phi_{i}}{z^{q}} d z\right\| \\
=\left\|\frac{\Delta_{n}^{q} T}{2 \pi} \int_{\Gamma} \frac{(T-z I)^{-1} \phi_{i}}{z^{q}} d z\right\| \leq \frac{c_{1} \ell(\Gamma)\left\|\phi_{i}\right\|}{2 \pi} \frac{\left\|\Delta_{n}^{q} T\right\|}{\epsilon^{q}} .
\end{aligned}
$$

Hence

$$
\left\|\left(\underline{\mathbf{T}}_{q}^{(n)}-\underline{\mathbf{T}}_{q, n}\right) \underline{\Phi}_{q}\right\|_{\infty} \leq \frac{c_{1} \ell(\Gamma)\|\phi\|_{\infty}}{2 \pi} \frac{\left\|\Delta_{n}^{q} T\right\|}{\epsilon^{q}}
$$

The bound for $\left\|\mathbf{P}_{q, n}\right\|_{\infty}$ is immediate. Also, since $\underline{\Phi}_{q} \in R\left(\underline{\mathbf{P}}_{q}\right)=R\left(\underline{\mathbf{P}}_{q}^{(n)}\right)$ for large enough $n$ by Proposition 2.1, we see that

$$
\begin{aligned}
\underline{\mathbf{P}}_{q, n} \underline{\Phi}_{q}-\underline{\Phi}_{q} & =\left(\underline{\mathbf{P}}_{q, n}-\underline{\mathbf{P}}_{q}^{(n)}\right) \underline{\Phi}_{q} \\
& =-\frac{1}{2 \pi i} \int_{\Gamma}\left(\underline{\mathbf{T}}_{q, n}-z \underline{\mathbf{I}}_{q}\right)^{-1}\left(\underline{\mathbf{T}}_{q}^{(n)}-\underline{\mathbf{T}}_{q, n}\right)\left(\underline{\mathbf{T}}_{q}^{(n)}-z \underline{\mathbf{I}}_{q}\right)^{-1} \underline{\Phi}_{q} d z \\
& =-\frac{1}{2 \pi i} \int_{\Gamma}\left(\underline{\mathbf{T}}_{q, n}-z \underline{\mathbf{I}}_{q}\right)^{-1}\left(\underline{\mathbf{T}}_{q}^{(n)}-\underline{\mathbf{T}}_{q, n}\right) \underline{\Phi}_{q}\left[\left(\underline{\mathbf{T}}_{q}^{(n)}-z \underline{\mathbf{I}}_{q}\right)^{-1} \underline{\Phi}_{q}, \underline{\Phi}_{q}^{*}\right] d z
\end{aligned}
$$

For $z \in \Gamma$, we have

$$
\begin{aligned}
\left\|\left[\left(\underline{\mathbf{T}}_{q}^{(n)}-z \underline{\mathbf{I}}_{q}\right)^{-1} \underline{\Phi}_{q}, \underline{\Phi}_{q}^{*}\right]\right\|_{1} & \leq\left\|\left(\underline{\mathbf{T}}_{q}^{(n)}-z \mathbf{I}_{q}\right)^{-1}\right\|_{\infty}\left\|\underline{\Phi}_{q}\right\|_{\infty}\left\|\underline{\Phi}_{q}^{*}\right\|_{1} \\
& \leq C_{3} \max \left\{1, \frac{\ell(\Gamma) c_{1}}{2 \pi}\right\}\|\phi\|_{\infty}\left\|\underline{\phi}^{*}\right\|_{1}
\end{aligned}
$$

Employing the bound for $\left\|\left(\underline{\mathbf{T}}_{q}^{(n)}-\underline{\mathbf{T}}_{q, n}\right) \Phi_{q}\right\|_{\infty}$ given earlier, we obtain the desired bound for $\left\|\underline{\mathbf{P}}_{q, n} \underline{\Phi}_{q}-\underline{\Phi}_{q}\right\|_{\infty}$.

By the definition of $\Sigma_{q, n}$ and $L_{q, n}$, we have

$$
\left\|\Sigma_{q, n}\right\|_{\infty} \leq \frac{\ell(\Gamma)}{2 \pi}\left(\max _{z \in \Gamma}\left\|\left(\mathbf{T}_{q, n}-z \mathbf{I}_{q}\right)^{-1}\right\|_{\infty}\right)^{2}\left\|\underline{\Phi}_{q, n}\right\|_{\infty}\left\|\Phi_{q, n}^{*}\right\|_{1} .
$$

Hence the bound for $\left\|\Sigma_{q, n}\right\|_{\infty}$ follows.

(b) We have

$$
\begin{aligned}
\left\|N_{q, n} M_{q, n}-I_{m}\right\|_{1} & =\left\|\left[\Phi_{q, n} M_{q, n}-\underline{\Phi}_{q}, \underline{\Phi}_{q}^{*}\right]\right\|_{1}=\left\|\left[\underline{\mathbf{P}}_{q, n} \underline{\Phi}_{q}-\underline{\Phi}_{q}, \underline{\Phi}_{q}^{*}\right]\right\|_{1} \\
& \leq\left\|\underline{\mathbf{P}}_{q, n} \underline{\Phi}_{q}-\underline{\Phi}_{q}\right\|_{\infty}\left\|\underline{\Phi}_{q}^{*}\right\|_{1} \leq C\left\|\underline{\phi}^{*}\right\|_{1} \frac{\left\|\Delta_{n}^{q} T\right\|}{\epsilon^{q}}
\end{aligned}
$$


by (a) above. If $\left\|\Delta_{n}^{2}\right\| \leq \epsilon^{2}$, then for all $q=2,3, \ldots$,

$$
\frac{\left\|\Delta_{n}^{q}\right\|}{\epsilon^{q}} \leq \max \left\{1, \frac{\left\|\Delta_{n}\right\|}{\epsilon}\right\} \frac{\left\|\Delta_{n}^{2}\right\|}{\epsilon^{2}} .
$$

As $\left(\left\|\Delta_{n}\right\|\right)$ is bounded and $\left\|\Delta_{n}^{2}\right\| \rightarrow 0$ as $n \rightarrow \infty$, it follows that $\left\|N_{q, n} M_{q, n}-I_{m}\right\|_{1} \rightarrow 0$ as $n \rightarrow \infty$, uniformly in $q=2,3, \ldots$.

We recall from [10, Theorem 3.2] that in the case of a simple eigenvalue, that is, when $m=1$, the initial choice of an eigenvector $\Phi_{q, n}$ of $\mathbf{T}_{q, n}$ corresponding to its simple eigenvalue $\lambda_{q, n}$ is made so that the first component $\phi_{q, n}$ of $\Phi_{q, n}$ satisfies

$$
0<d \leq\left\|\phi_{q, n}\right\| \leq c
$$

for some constants $c \geq d>0$, independent of $q$ and $n$. It was shown in [10, Section 4] that such a choice can be realized in practice when $T_{n}$ is a finite rank operator. In order to be able to implement a refinement scheme for a cluster $\Lambda$ of eigenvalues of $T$, we propose a normalization of a basis of $R\left(\mathbf{P}_{q, n}\right)$ (and not just of its first components), which is akin to orthonormalization of a linearly independent set in a Hilbert space.

THEOREM 3.2. Let $\min \{|\lambda|: \lambda \in \Lambda\}>\epsilon \geq 1$. Suppose that an ordered basis given by $\Phi_{q, n}=\left[\Phi_{q, n, 1}, \ldots, \Phi_{q, n, m}\right]$ of the spectral subspace $R\left(\mathbf{P}_{q, n}\right)$ associated with $\mathbf{T}_{q, n}$ and $\Lambda_{q, n}$ is so chosen that

(i) $\left\|\Phi_{q, n}\right\|_{\infty} \leq c$ and

(ii) for $i=1, \ldots, m, \operatorname{dist}\left(\Phi_{q, n, i}, \operatorname{span}\left\{\Phi_{q, n, k}: k=1, \ldots, m, k \neq i\right\}\right) \geq d>0$, for all large $n$, all $q=2,3, \ldots$ and some constants $c \geq d>0$ independent of $q$ and $n$.

Let $M_{q, n}=\left[\Phi_{q}, \underline{\Phi}_{q, n}^{*}\right], \Phi_{q,(n)}=\Phi_{q} M_{q, n}^{-1}$ and $L_{q,(n)}=\left[\underline{T}_{q}^{(n)} \Phi_{q,(n)}, \underline{\Phi}_{q, n}^{*}\right]$. Then $\left[\Phi_{q,(n)}, \Phi_{q, n}^{*}\right]=I_{m}$ and there is a positive integer $n_{1}$ such that for all $n \geq n_{1}$, all $q=2,3, \ldots$ and $j=0,1, \ldots$,

$$
\left\|L_{q,(n)}-L_{q, n}^{(j)}\right\|_{1},\left\|\Phi_{q,(n)}-\Phi_{q, n}^{(j)}\right\|_{\infty} \leq \frac{a}{\epsilon^{q}}\left\|\left(T-T_{n}\right)^{q} T\right\|\left\{b\left\|\left(T-T_{n}\right)^{q}\right\|\right\}^{j},
$$

for some constants $a$ and $b$, independent of $q, n$ and $j$.

PROOF. By part (b) of Lemma 3.1, the matrix $M_{q, n}$ is nonsingular for all large $n$ and all $q=2,3, \ldots$. Hence $\Phi_{q,(n)}$ is well-defined. Clearly

$$
\left[\Phi_{q,(n)}, \Phi_{q, n}^{*}\right]=\left[\Phi_{q}, \Phi_{q, n}^{*}\right] M_{q, n}^{-1}=M_{q, n} M_{q, n}^{-1}=I_{m} .
$$

Let us consider the sequences

$$
\left(\left\|M_{q, n}^{-1}\right\|_{1}\right), \quad\left(\left\|M_{q, n}\right\|_{1}\right), \quad\left(\left\|\Phi_{q, n}^{*}\right\|_{1}\right) \quad \text { and } \quad\left(\left\|\Sigma_{q, n}\right\|_{\infty}\right) .
$$


Since $N_{q, n} M_{q, n} \rightarrow I_{m}$ as $n \rightarrow \infty$, uniformly in $q=2,3, \ldots$ by part (b) of Lemma 3.1,

$$
\begin{aligned}
\left\|M_{q, n}^{-1}\right\|_{1} & =\left\|M_{q, n}^{-1} N_{q, n}^{-1} N_{q, n}\right\|_{1} \leq 2\left\|N_{q, n}\right\|_{1} \\
& =2\left\|\left[\underline{\Phi}_{q, n}, \underline{\Phi}_{q}^{*}\right]\right\|_{1} \leq 2\left\|\underline{\Phi}_{q, n}\right\|_{\infty}\left\|\underline{\Phi}_{q}^{*}\right\|_{1} \leq 2 c\left\|\underline{\phi}^{*}\right\|_{1} .
\end{aligned}
$$

Hence the sequence $\left(\left\|M_{q, n}^{-1}\right\|_{1}\right)$ is bounded uniformly in $q$ and $n$.

Next, $M_{q, n}=\left[\Phi_{q}, \Phi_{q, n}^{*}\right]$ with $\underline{\Phi}_{q}=\left[\Phi_{q, 1}, \ldots, \Phi_{q, m}\right]$ and

$$
\Phi_{q, n}^{*}=\left[\Phi_{q, n, 1}^{*}, \ldots, \Phi_{q, n, m}^{*}\right]
$$

so that

$$
\left\|M_{q, n}\right\|_{1}=\max _{j=1, \ldots, m} \sum_{i=1}^{m}\left|\left\langle\Phi_{q, j}, \Phi_{q, n, i}^{*}\right\rangle\right| .
$$

Now fix $j, 1 \leq j \leq m$. We have

$$
\mathbf{P}_{q, n} \Phi_{q, j}=\left\langle\Phi_{q, j}, \Phi_{q, n, 1}^{*}\right\rangle \Phi_{q, n, 1}+\cdots+\left\langle\Phi_{q, j}, \Phi_{q, n, m}^{*}\right\rangle \Phi_{q, n, m},
$$

so that for each fixed $i=1, \ldots, m$,

$$
\begin{aligned}
\left\|\mathbf{P}_{q, n} \Phi_{q, j}\right\|_{\infty} & \geq\left|\left\langle\Phi_{q, j}, \Phi_{q, n, i}^{*}\right\rangle\right| \operatorname{dist}\left(\Phi_{q, n, i}, \operatorname{span}\left\{\Phi_{q, n, k}: k=1, \ldots, m, k \neq i\right\}\right) \\
& \geq\left|\left\langle\Phi_{q, j}, \Phi_{q, n, i}^{*}\right\rangle\right| d
\end{aligned}
$$

by our assumption. Thus for each $i=1, \ldots, m$,

$$
d\left|\left\langle\Phi_{q, j}, \Phi_{q, n, i}^{*}\right\rangle\right| \leq\left\|\mathbf{P}_{q, n} \Phi_{q, j}\right\|_{\infty} \leq\left\|\mathbf{P}_{q, n}\right\|_{\infty}\left\|\Phi_{q, j}\right\|_{\infty} \leq\left\|\mathbf{P}_{q, n}\right\|_{\infty}\left\|\underline{\Phi}_{q}\right\|_{\infty}
$$

Hence

$$
\sum_{i=1}^{m}\left|\left\langle\Phi_{q, j}, \Phi_{q, n, i}^{*}\right\rangle\right| \leq \frac{m}{d}\left\|\mathbf{P}_{q, n}\right\|_{\infty}\left\|\Phi_{q}\right\|_{\infty} .
$$

Since $\left\|\mathbf{P}_{q, n}\right\|_{\infty} \leq \ell(\Gamma) C_{2} / 2 \pi$ and $\left(\left\|\Phi_{q}\right\|_{\infty}\right)$ is bounded uniformly in $q=2,3, \ldots$ by part (a) of Lemma 3.1, we see that the sequence $\left(\left\|M_{q, n}\right\|_{1}\right)$ is bounded uniformly in $q$ and $n$.

Further,

$$
\begin{aligned}
\left\|\underline{\Phi}_{q, n}^{*}\right\|_{1} & =\left\|\underline{\mathbf{P}}_{q, n}^{*} \underline{\Phi}_{q}^{*}\left(N_{q, n}^{H}\right)^{-1}\right\|_{1} \leq\left\|\underline{\mathbf{P}}_{q, n}^{*}\right\|_{1}\left\|\underline{\Phi}_{q}^{*}\right\|_{1}\left\|\left(N_{q, n}^{H}\right)^{-1}\right\|_{\infty} \\
& =\left\|\underline{\mathbf{P}}_{q, n}\right\|_{\infty}\left\|\underline{\Phi}_{q}^{*}\right\|_{1}\left\|N_{q, n}^{-1}\right\|_{1}=\left\|\underline{\mathbf{P}}_{q, n}\right\|_{\infty}\left\|\underline{\phi}^{*}\right\|_{1}\left\|M_{q, n} M_{q, n}^{-1} N_{q, n}^{-1}\right\|_{1} \\
& \leq \frac{\ell(\Gamma) C_{2}}{2 \pi}\left\|\underline{\Phi}^{*}\right\|_{1} 2\left\|M_{q, n}\right\|_{1} .
\end{aligned}
$$


Hence the sequence $\left(\left\|\Phi_{q, n}^{*}\right\|_{1}\right)$ is bounded uniformly in $q$ and $n$.

As the sequence $\left(\left\|\Phi_{q, n}\right\|_{\infty}\right)$ is also given to be uniformly bounded in $q$ and $n$, part (a) of Lemma 3.1 implies that the same holds for the sequence $\left(\left\|\Sigma_{q, n}\right\|_{\infty}\right)$.

Let us now consider the case $j=0$. We have

$$
\begin{aligned}
\left\|\Phi_{q,(n)}-\underline{\Phi}_{q, n}^{(0)}\right\|_{\infty} & =\left\|\Phi_{q} M_{q, n}^{-1}-\underline{\mathbf{P}}_{q, n} \Phi_{q} M_{q, n}^{-1}\right\|_{\infty} \\
& =\left\|\left(\Phi_{q}-\underline{\mathbf{P}}_{q, n} \Phi_{q}\right) M_{q, n}^{-1}\right\|_{\infty} \leq C \frac{\left\|\Delta_{n}^{q} T\right\|}{\epsilon^{q}} 2 c\left\|\underline{\phi}^{*}\right\|_{1}
\end{aligned}
$$

by part (a) of Lemma 3.1. Also,

$$
\begin{aligned}
L_{q,(n)}-L_{q, n}^{(0)} & =L_{q,(n)}-L_{q, n} \\
& =\left[\left(\underline{\mathbf{T}}_{q}^{(n)}-\underline{\mathbf{T}}_{q, n}\right) \underline{\Phi}_{q,(n)}, \underline{\Phi}_{q, n}^{*}\right]+\left[\underline{\mathbf{T}}_{q, n}\left(\Phi_{q,(n)}-\underline{\Phi}_{q, n}\right), \underline{\Phi}_{q, n}^{*}\right] .
\end{aligned}
$$

Since by part (a) of Lemma 3.1,

$$
\left\|\left(\underline{\mathbf{T}}_{q}^{(n)}-\underline{\mathbf{T}}_{q, n}\right) \Phi_{q,(n)}\right\|_{\infty}=\left\|\left(\underline{\mathbf{T}}_{q}^{(n)}-\underline{\mathbf{T}}_{q, n}\right) \underline{\Phi}_{q} M_{q, n}^{-1}\right\|_{\infty} \leq \frac{\ell(\Gamma) c_{1}\|\underline{\phi}\|_{\infty}}{2 \pi}\left\|M_{q, n}^{-1}\right\|_{1} \frac{\left\|\Delta_{n}^{q} T\right\|}{\epsilon^{q}}
$$

and since the sequences $\left(\left\|M_{q, n}^{-1}\right\|_{1}\right),\left(\left\|\Phi_{q, n}^{*}\right\|_{1}\right)$ and $\left(\left\|\underline{\mathbf{T}}_{q, n}\right\|\right)$ are bounded uniformly in $q$ and $n$, we see that there is a positive integer $n_{0}$ such that for all $n \geq n_{0}$ and all $q=2,3, \ldots$,

$$
\left\|\Phi_{q,(n)}-\Phi_{q, n}^{(0)}\right\|_{\infty},\left\|L_{q,(n)}-L_{q, n}^{(0)}\right\|_{1} \leq \frac{a}{\epsilon^{q}}\left\|\Delta_{n}^{q} T\right\|,
$$

for some constant $a$ independent of $q$ and $n$.

As $\left\|\Phi_{q,(n)}\right\|_{\infty} \leq\left\|\Phi_{q}\right\|_{\infty}\left\|M_{q, n}^{-1}\right\|_{1}$, we see that the sequence $\left(\left\|\Phi_{q,(n)}\right\|_{\infty}\right)$ is bounded uniformly in $q$ and $n$. Let $b$ be a positive constant such that

$$
\left\|\underline{\Phi}_{q, n}^{*}\right\|_{1} \leq b \text { and }\left\|\Sigma_{q, n}\right\|_{\infty}\left(1+\left\|\underline{\Phi}_{q,(n)}\right\|_{\infty}\left\|\Phi_{q, n}^{*}\right\|_{1}+2 a\|T\|\right) \leq b
$$

for all large $n$ and all $q=2,3, \ldots$. Since $\left\|\mathbf{T}_{q}^{(n)}-\mathbf{T}_{q, n}\right\|_{\infty}=\left\|\left(T-T_{n}\right)^{q}\right\| \rightarrow 0$ as $n \rightarrow \infty$, uniformly in $q=2,3, \ldots$, there is a positive integer $n_{1}$ such that $\left\|\left(T-T_{n}\right)^{q}\right\| \leq 1 / b$ for all $n \geq n_{1}$ and all $q=2,3, \ldots$. The rest of the proof is the same as given in [10, Theorem 3.2].

COROLLARY 3.3. Let the assumptions and notations of Theorem 3.2 hold. Let $\hat{\lambda}$ denote the arithmetic mean of the eigenvalues in $\Lambda$, counted according to their algebraic multiplicities, and let $\underline{\phi}=\left[\phi_{1}, \ldots, \phi_{m}\right]$ give a basis of the corresponding spectral subspace of T. Define

$$
\hat{\lambda}_{q, n}^{(j)}=\frac{1}{m} \operatorname{trace} L_{q, n}^{(j)}, \quad j=0,1, \ldots,
$$


and let $\underline{\phi}_{q, n}^{(j)}$ denote the first row of $\Phi_{q, n}^{(j)}$. Then for all large $n$, all $q=2,3, \ldots$, and $j=0, \overline{1}, 2, \ldots$

$$
\left|\hat{\lambda}-\hat{\lambda}_{q, n}^{(j)}\right|,\left\|\underline{\phi} M_{q, n}^{-1}-\underline{\phi}_{q, n}^{(j)}\right\|_{\infty} \leq \frac{a}{\epsilon^{q}}\left\|\left(T-T_{n}\right)^{q} T\right\|\left\{b\left\|\left(T-T_{n}\right)^{q}\right\|\right\}^{j}
$$

for some constants $a$ and $b$, independent of $q, n$ and $j$.

Proof. Noting that $\min \{|\lambda|: \lambda \in \Lambda\}>1$, we obtain $R\left(\mathbf{P}_{q}\right)=R\left(\mathbf{P}_{q}^{(n)}\right)$ by Proposition 2.1 for all large $n$ and all $q=2,3, \ldots$, for which we also have

$$
\sigma\left(\mathbf{T}_{q}^{(n)}\right) \cap \operatorname{Int} \Gamma=\sigma\left(\mathbf{T}_{q}\right) \cap \operatorname{Int} \Gamma=\Lambda
$$

Hence $L_{q,(n)}=\left[\underline{\mathbf{T}}_{q}^{(n)} \underline{\Phi}_{q,(n)}, \Phi_{q, n}^{*}\right]$ is the matrix representation of the operator $\left.\mathbf{T}_{q}^{(n)}\right|_{R\left(\mathbf{P}_{q}^{(n)}\right)}$ with respect to the ordered basis given by $\Phi_{q,(n)}$ and $\hat{\lambda}=(1 / m)$ trace $L_{q,(n)}$. Thus

$$
\left|\hat{\lambda}-\hat{\lambda}_{q, n}^{(j)}\right| \leq\left\|L_{q,(n)}-L_{q, n}^{(j)}\right\|_{1}
$$

Also, since $\underline{\phi} M_{q, n}^{-1}$ is the first row of $\underline{\Phi}_{q,(n)}$ and

$$
\left\|\underline{\phi} M_{q, n}^{-1}-\underline{\phi}_{q, n}^{(j)}\right\|_{\infty} \leq\left\|\underline{\Phi}_{q,(n)}-\underline{\Phi}_{q, n}^{(j)}\right\|_{\infty}
$$

we obtain the desired results from Theorem 3.2.

In order to treat the case when $\min \{|\lambda|: \lambda \in \Lambda\}>\epsilon$ and $0<\epsilon<1$, we let $\alpha=1 / \epsilon, \tilde{\Lambda}=\{\alpha \lambda: \lambda \in \Lambda\}$ and consider the scaled operators $\tilde{\mathbf{T}}_{q}, \tilde{\mathbf{T}}_{q, n}, \tilde{\mathbf{T}}_{q}^{(n)}$ and the corresponding spectral projections $\tilde{\mathbf{P}}_{q}, \tilde{\mathbf{P}}_{q, n}, \tilde{\mathbf{P}}_{q}^{(n)}$, respectively as given in [9, Section 3] and [10, Section 3].

Note that if $D_{q}: \mathbf{X}_{q} \rightarrow \mathbf{X}_{q}$ is given by

$$
D_{q}\left[x_{1}, \ldots, x_{q}\right]^{t}=\left[x_{1}, \alpha x_{2}, \ldots, \alpha^{q-1} x_{q}\right]^{t}, \quad\left[x_{1}, \ldots, x_{q}\right]^{t} \in \mathbf{X}_{q} \text {, }
$$

then $\underline{\Phi}_{q}$ gives a basis of $R\left(\mathbf{P}_{q}\right)$ if and only if $\tilde{\Phi}_{q}=\underline{D}_{q}^{-1} \Phi_{q}$ gives a basis of $R\left(\tilde{\mathbf{P}}_{q}\right)$, and $\Phi_{q}^{*}$ gives a basis of $R\left(\mathbf{P}_{q}^{*}\right)$ if and only if $\tilde{\Phi}_{q}^{*}=\underline{D}_{q} \underline{\Phi}_{q}^{*}$ gives a basis of $R\left(\tilde{\mathbf{P}}_{q}^{*}\right)$. Also, $\left[\tilde{\Phi}_{q}, \tilde{\Phi}_{q}^{*}\right]=\left[\Phi_{q}, \Phi_{q}^{*}\right]$. Similar results hold for $R\left(\mathbf{P}_{q, n}\right)$ and $R\left(\mathbf{P}_{q}^{(n)}\right)$. If $\Sigma_{q, n}$ is the block-reduced resolvent associated with $\mathbf{T}_{q, n}$ and $\Lambda_{q, n}$ with respect to the basis $\Phi_{q, n}$, and $\tilde{\Sigma}_{q, n}$ is the block-reduced resolvent associated with $\tilde{\mathbf{T}}_{q, n}$ and $\tilde{\Lambda}_{q, n}$ with respect to the basis $\tilde{\Phi}_{q, n}$, then it follows that

$$
\tilde{\Sigma}_{q, n}=\underline{D}_{q}^{-1} \frac{\Sigma_{q, n}}{\alpha} \underline{D}_{q}
$$

Also, let $\underline{\Phi}_{q, n}^{*}=\underline{D}_{q} \underline{\Phi}_{q, n}^{*}$. 
Consider the fixed-slope Newton scheme corresponding to the scaled operators:

$$
\begin{aligned}
& \tilde{L}_{q, n}^{(0)}:=\alpha L_{q, n}, \quad \tilde{\Phi}_{q, n}^{(0)}:=\underline{D}_{q}^{-1} \Phi_{q, n} \text { and for } j=1,2, \ldots, \\
& \tilde{L}_{q, n}^{(j)}:=\left[\tilde{\mathbf{T}}_{q}^{(n)} \tilde{\Phi}_{q, n}^{(j-1)}, \tilde{\Phi}_{q, n}^{*}\right], \\
& \tilde{\Phi}_{q, n}^{(j)}:=\tilde{\Phi}_{q, n}^{(j-1)}-\tilde{\Sigma}_{q, n}\left[\tilde{\mathbf{T}}_{q}^{(n)} \tilde{\Phi}_{q, n}^{(j-1)}-\underline{\Phi}_{q, n}^{(j-1)} \tilde{L}_{q, n}^{(j)}\right] .
\end{aligned}
$$

In exactly the same way as in [10, Lemma 3.3], we see that

$$
\tilde{L}_{q, n}^{(j)}=\alpha L_{q, n}^{(j)} \quad \text { and } \quad \tilde{\Phi}_{q, n}^{(j)}=\underline{D}_{q}^{-1} \Phi_{q, n}^{(j)}
$$

for $j=0,1, \ldots$ In particular, the first row of the scaled iterate $\tilde{\Phi}_{q, n}^{(j)}$ is the same as the first row of the iterate $\Phi_{q, n}^{(j)}$.

Hence we obtain error estimates similar to those given in Theorem 3.2 and Corollary 3.3 if $\min \{|\lambda|: \lambda \in \Lambda\}>\epsilon$, where the positive number $\epsilon$ may be less than 1 .

\section{Implementation and numerical examples}

In this section we show how the refinement scheme (2.1) can be implemented when each $T_{n}$ is a bounded operator of finite rank and we illustrate this procedure by considering multiple eigenvalues of an integral operator. We also demonstrate how a basis of the spectral subspace associated with $\mathbf{T}_{q, n}$ and $\Lambda_{q, n}$ can be chosen so that each element is bounded and is bounded away from all other elements as required in Theorem 3.2.

As in [10], we assume that the rank of $T_{n} \leq n$ and

$$
T_{n} x=\left\langle x, x_{n, 1}^{*}\right\rangle x_{n, 1}+\cdots+\left\langle x, x_{n, n}^{*}\right\rangle x_{n, n}, \quad x \in X .
$$

We use the maps $F_{n}, G_{n}$ and $\mathbf{F}_{q, n}$ and the matrix $\mathbf{A}_{q, n}$ introduced in [10, Section 4]. Further, define $\underline{F}_{n}: \underline{X} \rightarrow \mathbb{C}^{n \times m}$ and $\underline{G}_{n}: \mathbb{C}^{n \times m} \rightarrow \underline{X}$ by

$$
\begin{array}{ll}
\underline{F}_{n} \underline{x}=\left[F_{n} x_{1}, \ldots, F_{n} x_{m}\right], & \underline{x}=\left[x_{1}, \ldots, x_{m}\right] \in \underline{X}, \\
\underline{G}_{n} \underline{u}=\left[G_{n} u_{1}, \ldots, G_{n} u_{m}\right], & \underline{u}=\left[u_{1}, \ldots, u_{m}\right] \in \mathbb{C}^{n \times m} .
\end{array}
$$

We have seen in [10] that the nonzero eigenvalues of the finite rank operator $\mathbf{T}_{q, n}$ and of the $q n \times q n$ matrix $\mathbf{A}_{q, n}$ are the same. Thus we choose a set $\Lambda_{q, n}$ of nonzero eigenvalues of $\mathbf{A}_{q, n}$ whose total algebraic multiplicity is $m$ and which is supposed to approximate the cluster $\Lambda$ of nonzero eigenvalues of $T$. Let $U_{q, n, 1}, \ldots, U_{q, n, m} \in$ $\mathbb{C}^{q n}$ constitute an ordered basis of the spectral subspace of $\mathbf{A}_{q, n}$ associated with its 
eigenvalues belonging to $\Lambda_{q, n}$. Since this subspace of $\mathbb{C}^{q n}$ is invariant under $\mathbf{A}_{q, n}$, we see that if $\underline{U}_{q, n}=\left[U_{q, n, 1}, \ldots, U_{q, n, m}\right] \in \mathbb{C}^{q n \times m}$, then

$$
\mathbf{A}_{q, n} \underline{U}_{q, n}=\underline{U}_{q, n} \Theta_{q, n}
$$

for some $m \times m$ matrix $\Theta_{q, n}$ with complex entries. Since $0 \notin \Lambda_{q, n}$, the matrix $\Theta_{q, n}$ is invertible. Next, there is a unique ordered basis $V_{q, n, 1}, \ldots, V_{q, n, m}$ of the spectral subspace of $A_{q, n}^{H}$ associated with $\left\{\bar{\lambda}_{q, n}: \lambda_{q, n} \in \Lambda_{q, n}\right\}$ such that

$$
\left\langle U_{q, n, j}, V_{q, n, i}\right\rangle=V_{q, n, i}^{H} U_{q, n, j}=\delta_{i, j}, \quad i, j=1, \ldots, m .
$$

If $\underline{V}_{q, n}=\left[V_{q, n, 1}, \ldots, V_{q, n, m}\right] \in \mathbb{C}^{q n \times m}$, then

$$
\mathbf{A}_{q, n}^{H} \underline{V}_{q, n}=\underline{V}_{q, n} \Theta_{q, n}^{H} \quad \text { and } \quad\left[\underline{U}_{q, n}, \underline{V}_{q, n}\right]=I_{q n}
$$

Hence

$$
\Theta_{q, n}=\left[\mathbf{A}_{q, n} \underline{U}_{q, n}, \underline{V}_{q, n}\right]
$$

If

$$
\underline{U}_{q, n}=\left[\begin{array}{c}
\underline{u}_{1} \\
\vdots \\
\underline{u}_{q}
\end{array}\right] \in \mathbb{C}^{q n \times m},
$$

where $\underline{u}_{1} \in \mathbb{C}^{n \times m}$ denotes the first $n$ rows of $\underline{U}_{q, n}, \underline{u}_{2} \in \mathbb{C}^{n \times m}$ denotes the next $n$ rows of $\underline{U}_{q, n}$ and so on, define

$$
\underline{\Phi}_{q, n}=\left[\sum_{j=0}^{q-1}\left(\underline{\Delta}_{n}^{j} \underline{G}_{n} \underline{u}_{1}\right) \Theta_{q, n}^{-j-1}, \ldots, \sum_{j=0}^{q-1}\left(\underline{\Delta}_{n}^{j} \underline{G}_{n} \underline{u}_{q}\right) \Theta_{q, n}^{-j-1}\right]^{t}
$$

and

$$
\underline{\Phi}_{q, n}^{*}=\underline{\mathbf{F}}_{q, n}^{*} \underline{V}_{q, n}
$$

Let $\Phi_{q, n}=\left[\Phi_{q, n, 1}, \ldots, \Phi_{q, n, m}\right]$. Then it can be seen that $\Phi_{q, n, 1}, \ldots, \Phi_{q, n, m}$ constitute an ordered basis of the spectral subspace $R\left(\mathbf{P}_{q, n}\right)$ of $\mathrm{T}_{q, n}$ associated with $\Lambda_{q, n}$ and

$$
\underline{\mathbf{F}}_{q, n} \underline{\Phi}_{q, n}=\mathbf{A}_{q, n} \underline{U}_{q, n} \Theta_{q, n}^{-1}=\underline{U}_{q, n},
$$

so that

$$
\left[\Phi_{q, n}, \underline{\Phi}_{q, n}^{*}\right]=\left[\underline{\Phi}_{q, n}, \underline{\mathbf{F}}_{q, n}^{*} \underline{V}_{q, n}\right]=\left[\underline{\mathbf{F}}_{q, n} \underline{\Phi}_{q, n}, \underline{V}_{q, n}\right]=\left[\underline{U}_{q, n}, \underline{V}_{q, n}\right]=I_{m}
$$


In fact, if $\Phi_{q, n}^{*}=\left[\Phi_{q, n, 1}^{*}, \ldots, \Phi_{q, n, m}^{*}\right]$, then $\Phi_{q, n, 1}^{*}, \ldots, \Phi_{q, n, m}^{*}$ constitute the unique ordered basis of $R\left(\mathbf{P}_{q}^{*} n\right)$ satisfying $\left[\Phi_{q, n}, \Phi_{q, n}^{*}\right]=I_{m}$. It is easy to see that

$$
\underline{\mathbf{T}}_{q, n} \underline{\Phi}_{q, n}=\underline{\Phi}_{q, n} \Theta_{q, n} \quad \text { and } \quad \underline{\mathbf{T}}_{q, n}^{*} \underline{\Phi}_{q, n}^{*}=\underline{\Phi}_{q, n}^{*} \Theta_{q, n}^{H} .
$$

(Compare the case of a simple eigenvalue, that is, when $m=1$, treated in [10].)

Let \|\|$_{n}$ be a norm on $\mathbb{C}^{n}$ such that $\left\|F_{n}\right\| \leq \alpha$ and $\left\|G_{n}\right\| \leq \beta$ for some constants $\alpha$ and $\beta$ independent of $n$. We remark that the boundedness of the sequences $\left(\left\|F_{n}\right\|\right)$ and $\left(\left\|G_{n}\right\|\right)$ depends on the choice of a norm \|\|$_{n}$ on $\mathbb{C}^{n}$ and, in general, this choice will be dictated by the given norm \|\| on the Banach space $X$. We refer to [8] for various examples of this kind.

$$
\text { For } U=\left[\begin{array}{c}
u_{1} \\
\vdots \\
u_{q}
\end{array}\right] \in \mathbb{C}^{q n} \text { with } u_{1}, \ldots, u_{q} \text { in } \mathbb{C}^{n} \text {, let }\|U\|_{\infty}=\max \left\{\left\|u_{1}\right\|_{n}, \ldots,\left\|u_{q}\right\|_{n}\right\}
$$

and for $\underline{U}=\left[U_{1}, \ldots, U_{m}\right] \in \mathbb{C}^{q n \times m}$, let $\|\underline{U}\|_{\infty}=\max \left\{\left\|U_{1}\right\|_{\infty}, \ldots,\left\|U_{m}\right\|_{\infty}\right\}$.

THEOREM 4.1. Suppose that the sequence $\left(\left\|\underline{U}_{q, n}\right\|_{\infty}\right)$ is bounded uniformly in $q$ and $n$, and for all large $n$, all $q=2,3, \ldots$ and all $i=1, \ldots, m$,

$$
\operatorname{dist}\left(U_{q, n, i}, \operatorname{span}\left\{U_{q, n, k}: k=1, \ldots, m, k \neq i\right\}\right) \geq \delta
$$

for some constant $\delta>0$. Then

(i) the sequence $\left(\left\|\Phi_{q, n}\right\|_{\infty}\right)$ is bounded uniformly in $q$ and $n$; and

(ii) for all $i=1, \ldots, m, \operatorname{dist}\left(\Phi_{q, n, i}, \operatorname{span}\left\{\Phi_{q, n, k}: k=1, \ldots, m, k \neq i\right\}\right) \geq d$ for some constant $d>0$.

PROOF. Recall that $\left\|F_{n}\right\| \leq \alpha$ and $\left\|G_{n}\right\| \leq \beta$. Let $\left\|\underline{U}_{q, n}\right\|_{\infty} \leq C$ for all large $n$ and all $q=2,3, \ldots$. Choose $n$ so large that $\left\|\Delta_{n}^{2}\right\| \leq 1 / 2$. Then

$$
\begin{aligned}
\left\|\mathbf{A}_{q, n}\right\|_{\infty} & =\max \left\{1, \sum_{j=0}^{q-1}\left\|\Delta_{n}^{j}\right\|\left\|F_{n}\right\|\left\|G_{n}\right\|\right\} \leq \max \left\{1, \alpha \beta \frac{1+\left\|\Delta_{n}\right\|}{1-\left\|\Delta_{n}^{2}\right\|}\right\} \\
& \leq \max \{1,2 \alpha \beta(1+t)\},
\end{aligned}
$$

where $\left\|\Delta_{n}\right\| \leq t$ for all $n$. Let $\xi=\max \{1,2 \alpha \beta(1+t)\}$. First we find a bound for $\left\|\Theta_{q, n}\right\|_{1}$. Let $\theta_{i, j}$ denote the $(i, j)$ th entry of $\Theta_{q, n}$. Since $\mathbf{A}_{q, n} \underline{U}_{q, n}=\underline{U}_{q, n} \Theta_{q, n}$, we see that for each fixed $j=1, \ldots, m$,

$$
\mathbf{A}_{q, n} U_{q, n, j}=\theta_{1, j} U_{q, n, 1}+\cdots+\theta_{m, j} U_{q, n, m},
$$

and hence

$$
\delta\left|\theta_{i, j}\right| \leq\left\|\mathbf{A}_{q, n} U_{q, n, j}\right\|_{\infty} \leq \xi C .
$$


Thus

$$
\sum_{i=1}^{m}\left|\theta_{i, j}\right| \leq \frac{m \xi C}{\delta}
$$

showing that $\left\|\Theta_{q, n}\right\|_{1} \leq m \xi C / \delta=\gamma$, say. Let

$$
\min \left\{|\mu|: \mu \in \sigma\left(\Theta_{q, n}\right)\right\}>\epsilon
$$

for all large $n$ and all $q=2,3, \ldots$ Then Lemma 4.2 of [8] shows that for all large $n$ and all $q=2,3, \ldots$,

$$
\left\|\Theta_{q, n}^{-1}\right\|_{1} \leq \frac{m\left(m^{m} \gamma^{m}-\epsilon^{m}\right)}{(m \gamma-\epsilon) \epsilon^{m}}=\eta, \text { say. }
$$

Hence

$$
\left\|\underline{\Phi}_{q, n}\right\|_{\infty} \leq \sum_{j=0}^{q-1}\left\|\Delta_{n}^{j}\right\|\left\|G_{n}\right\|\left\|U_{q, n}\right\|_{\infty} \eta^{j+1} \leq \beta C \eta \sum_{j=0}^{q-1}\left\|\Delta_{n}^{j}\right\| \eta^{j}
$$

and for all large $n$ with $\left\|\Delta_{n}^{2}\right\| \leq 1 /\left(2 \eta^{2}\right)$, we have

$$
\left\|\underline{\Phi}_{q, n}\right\|_{\infty} \leq \beta C \eta \frac{1+\eta\left\|\Delta_{n}\right\|}{1-\eta^{2}\left\|\Delta_{n}^{2}\right\|} \leq 2 \beta C \eta(1+\eta t) .
$$

Thus the sequence $\left(\left\|\Phi_{q, n}\right\|_{\infty}\right)$ is bounded uniformly in $q$ and $n$.

For each fixed $i=1, \ldots, m$, and $a_{k} \in \mathbb{C}$ for $k=1, \ldots, m, k \neq i$, we have

$$
\begin{aligned}
\delta & \leq\left\|U_{q, n, i}-a_{1} U_{q, n, 1}-\cdots-a_{m} U_{q, n, m}\right\|_{\infty} \\
& =\left\|\mathbf{F}_{q, n}\left(\Phi_{q, n, i}-a_{1} \Phi_{q, n, 1}-\cdots-a_{m} \Phi_{q, n, m}\right)\right\|_{\infty} \\
& \leq \alpha\left\|\Phi_{q, n, i}-a_{1} \Phi_{q, n, 1}-\cdots-a_{m} \Phi_{q, n, m}\right\|_{\infty},
\end{aligned}
$$

so that

$$
\operatorname{dist}\left(\Phi_{q, n, i}, \operatorname{span}\left\{\Phi_{q, n, k}: k=1, \ldots, m, k \neq i\right\}\right) \geq \frac{\delta}{\alpha}=d>0
$$

Consider the case when $\min \{|\lambda|: \lambda \in \Lambda\}>1$. In view of the preceding result, it is clear that the assumptions in Theorem 3.2 can be realized in practice if we can find a basis $U_{q, n, 1} \cdots U_{q, n, m}$ of the spectral subspace of $\mathbf{A}_{q, n}$ associated with $\Lambda_{q, n}$ which satisfies the assumptions of Theorem 4.1 .

In the case when $\min \{|\lambda|: \lambda \in \Lambda\}>\epsilon$ with $0<\epsilon<1$, we need to consider the scaled operators $\tilde{\mathbf{T}}_{q, n}$ and $\tilde{\mathbf{T}}_{q}^{(n)}$ with $\alpha=1 / \epsilon$. If the scaled basis given by $\underline{\tilde{U}}_{q, n}=\underline{D}_{q}^{-1} \underline{U}_{q, n}$ satisfies the assumptions of Theorem 4.1 , then for the scaled basis given by $\underline{\Phi}_{q, n}=\underline{D}_{q}^{-1} \underline{\Phi}_{q, n}$, we have 
(i) $\left\|\tilde{\Phi}_{q, n}\right\|_{\infty} \leq \tilde{c}$; and

(ii) for each $i=1, \ldots, m, \operatorname{dist}\left(\tilde{\Phi}_{q, n, i}, \operatorname{span}\left\{\tilde{\Phi}_{q, n, k}: k=1, \ldots, m, k \neq i\right\}\right) \geq$ $\tilde{d}>0$

for all large $n$, all $q=2,3, \ldots$ and some constants $\tilde{c} \geq \tilde{d}>0$, independent of $q$ and $n$, as desired. Thus in this case we need to have a normalized scaled basis $\underline{\tilde{U}}_{q, n}=\underline{D}_{q}^{-1} \underline{U}_{q, n}$. For obtaining such a basis, the only additional requirement is that a lower bound $\epsilon$ for $\min \{|\lambda|: \lambda \in \Lambda\}$ should be available $a$ priori.

Starting with any basis of the spectral subspace of $\mathbf{A}_{q, n}$ associated with $\Lambda_{q, n}$, we can construct a basis $\left\{U_{q, n, 1}, \ldots, U_{q, n, m}\right\}$ of this subspace satisfying the assumptions of Theorem 4.1 as indicated in [8, Theorem 6.2, Section 4].

To illustrate the implementation procedure given above, we consider the space $X=C([a, b])$ of all complex-valued continuous functions on the interval $[a, b]$ with the sup norm. Let $T$ be the integral operator on $X$ given by

$$
T x(s)=\int_{a}^{b} k(s, t) x(t) d t, \quad x \in X, \quad s \in[a, b],
$$

where the kernel $k$ is continuous on $[a, b] \times[a, b]$. Note that $T$ is a compact operator on $X$. In actual computations, $T$ is replaced by its Nystrom approximation $\tilde{T}$ given by

$$
\tilde{T} x(s)=\sum_{j=1}^{M} w_{j}^{(M)} k\left(s, t_{j}^{(M)}\right) x\left(t_{j}^{(M)}\right), \quad x \in X, \quad s \in[a, b]
$$

where $M$ is very large. Here the nodes $t_{1}^{(M)}, \ldots, t_{j}^{(M)}$ in $[a, b]$ and the weights $w_{1}^{(M)}, \ldots, w_{j}^{(M)}$ in $\mathbb{C}$ are assumed to give a convergent quadrature formula

$$
Q x=\sum_{j=1}^{M} w_{j}^{(M)} x\left(t_{j}^{(M)}\right), \quad x \in X
$$

Thus in practice, a nonzero eigenvalue $\lambda$ of $T$ having algebraic multiplicity $m$ is replaced by a nearby cluster of nonzero eigenvalues of $\tilde{T}$ having total algebraic multiplicity $m$.

Consider the kernel $k:[0,1] \times[0,1] \rightarrow \mathbb{R}$ given by

$$
k(s, t)=\left\{\begin{array}{lll}
s-t / 2, & \text { if } & 0 \leq s \leq t \leq 1 \\
t / 2, & \text { if } & 0 \leq t<s \leq 1
\end{array}\right.
$$

Then for each $j=1,2, \ldots, 1 /(2 j-1)^{2} \pi^{2}$ is an eigenvalue of $T$ of algebraic multiplicity $m=2$ and ascent $l=2$. We use the same finite rank approximation $T_{n}$ of $T$ as in [10, Section 5]. 
We take $M=500$ and give numerical results for the largest and the second largest eigenvalues of $T$. The initial basis is normalized as required in Theorem 3.2. Let $\lambda$ denote the largest eigenvalue $1 / \pi^{2}$ of $T$. Also, let

$$
\hat{\lambda}_{q, n}^{(j)}=\frac{1}{m} \operatorname{trace} L_{q, n}^{(j)} \quad \text { and } \quad r_{q, n}^{(j)}=\left\|\underline{T} \underline{\phi}_{q, n}^{(j)}-\underline{\phi}_{q, n}^{(j)} L_{q, n}^{(j)}\right\|_{\infty}
$$

where $L_{q, n}^{(j)}$ and $\underline{\phi}_{q, n}^{(j)}$ are obtained from the $q$ th order fixed-slope Newton scheme. Let $\mu$ denote the second largest eigenvalue $1 / 9 \pi^{2}$ of $T$ and $\hat{\mu}_{q, n}^{(j)}$ and $s_{q, n}^{(j)}$ denote the corresponding quantities for $\mu$. The following computations were performed on CDC CYBER-180/840 with an accuracy of 15 digits.

For the implementation of the $q$ th order scheme for a cluster of eigenvalues of total algebraic multiplicity $m$, the size of the matrix eigenvalue problem solved for initialization is $q n$. Also, in each iteration a Sylvester equation of the form

$$
A W-W L=C
$$

is solved, where $A$ and $L$ are matrices of order $q n \times q n$ and $m \times m$, respectively.

TABLE 4.1. Error estimates for $q=1, n=30(q n=30)$

\begin{tabular}{|c|cc|cc|}
\hline$j$ & $\left|\lambda-\hat{\lambda}_{1,30}^{(j)}\right|$ & $r_{1,30}^{(j)}$ & $\left|\mu-\hat{\mu}_{1,30}^{(j)}\right|$ & $s_{1,30}^{(j)}$ \\
\hline 0 & $9.85 \times 10^{-5}$ & $7.20 \times 10^{-2}$ & $9.79 \times 10^{-5}$ & $1.24 \times 10^{-1}$ \\
1 & $3.10 \times 10^{-8}$ & $9.01 \times 10^{-4}$ & $2.74 \times 10^{-7}$ & $1.43 \times 10^{-3}$ \\
2 & $9.17 \times 10^{-12}$ & $1.17 \times 10^{-7}$ & $7.25 \times 10^{-10}$ & $1.54 \times 10^{-5}$ \\
3 & $6.22 \times 10^{-15}$ & $1.25 \times 10^{-10}$ & $6.29 \times 10^{-12}$ & $1.63 \times 10^{-7}$ \\
4 & & & $4.68 \times 10^{-14}$ & $1.69 \times 10^{-9}$ \\
5 & & & $1.28 \times 10^{-14}$ & $1.67 \times 10^{-10}$ \\
\hline
\end{tabular}

TABLE 4.2. Error estimates for $q=2$ and $3, n=5$ ( $q n=10$ and 15)

\begin{tabular}{|c|cc|cc|}
\hline$j$ & $\left|\lambda-\hat{\lambda}_{2,5}^{(j)}\right|$ & $r_{2,5}^{(j)}$ & $\left|\lambda-\hat{\lambda}_{3,5}^{(j)}\right|$ & $r_{3,5}^{(j)}$ \\
\hline 0 & $1.56 \times 10^{-4}$ & $9.93 \times 10^{-3}$ & $8.03 \times 10^{-6}$ & $3.32 \times 10^{-4}$ \\
1 & $1.11 \times 10^{-7}$ & $2.12 \times 10^{-5}$ & $1.04 \times 10^{-9}$ & $7.46 \times 10^{-8}$ \\
2 & $1.06 \times 10^{-10}$ & $3.68 \times 10^{-8}$ & $1.95 \times 10^{-13}$ & $2.43 \times 10^{-11}$ \\
3 & $9.86 \times 10^{-14}$ & $1.06 \times 10^{-10}$ & & \\
\hline
\end{tabular}

Table 4.1 gives error estimates for $q=1$ and $n=30$. The size of the matrix eigenvalue problem solved for initialization as well as the size of the coefficient matrix $A$ in the Sylvester equation solved in each iteration is $q n=30$.

Table 4.2 gives results for the largest eigenvalue $\lambda$ when $n=5$, and $q=2$ and 3 . Here the eigenvalue problems solved are of sizes $q n=10$ and 15 , respectively. 
TABLE 4.3. Error estimates for $q=2$ and $3, n=10(q n=20$ and 30)

\begin{tabular}{|c|cc|cc|}
\hline$j$ & $\left|\mu-\hat{\mu}_{2,10}^{(j)}\right|$ & $s_{2,10}^{(j)}$ & $\left|\mu-\hat{\mu}_{3,10}^{(j)}\right|$ & $s_{3,10}^{(j)}$ \\
\hline 0 & $7.76 \times 10^{-5}$ & $2.17 \times 10^{-2}$ & $8.36 \times 10^{-6}$ & $2.72 \times 10^{-3}$ \\
1 & $1.12 \times 10^{-7}$ & $1.25 \times 10^{-4}$ & $5.59 \times 10^{-9}$ & $3.21 \times 10^{-6}$ \\
2 & $5.46 \times 10^{-10}$ & $6.93 \times 10^{-7}$ & $3.95 \times 10^{-12}$ & $2.87 \times 10^{-9}$ \\
3 & $4.95 \times 10^{-12}$ & $1.21 \times 10^{-8}$ & & \\
4 & $1.11 \times 10^{-14}$ & $7.87 \times 10^{-11}$ & & \\
\hline
\end{tabular}

Table 4.3 gives results for the second largest eigenvalue $\mu$ when $n=10$, and $q=2$ and 3. Here the eigenvalue problems solved are of sizes $q n=20$ and 30 , respectively.

From these tables, it can be seen that employing higher order refinement schemes can be quite competitive.

\section{References}

[1] M. Ahues, "A superlinearly convergent two-grid defect correction method for invariant subspaces of compact operators", Numer. Funct. Anal. Optimiz. 8 (1985-86) 599-621.

[2] M. Ahues, S. Aranchiba and M. Telias, "Rayleigh-Schrödinger series for defective spectral elements of compact operators in Banach spaces. First part: Theoretical aspects", Numer. Funct. Anal. Optimiz. 11 (1990-91) 839-850.

[3] M. Ahues and F. Chatelin, "The use of defect correction to refine the eigenelements of compact integral operators", SIAM J. Numer. Anal. 20 (1983) 1087-1093.

[4] M. Ahues and A. Largillier, "Rayleigh-Schrödinger series for defective spectral elements of compact operators in Banach spaces. Second part: Numerical comparison with some inexact Newton methods", Numer. Funct. Anal. Optimiz. 11 (1990-91) 851-872.

[5] M. Ahues and A. Largillier, "Rayleigh-Schrödinger series versus inexact Newton methods for spectral computations", in Iterative methods in linear algebra (eds. R. Beauwens and P. de Groen), (Elsevier Science Publishers B.V. North Holland, 1992) 415-422.

[6] M. Ahues and A. Largillier, "A variant of fixed tangent method for spectral computations on integral operators", Numer. Funct. Anal. Optimiz. 16 (1995) 1-17.

[7] M. Ahues and M. Telias, "Refinement methods of Newton type for approximate eigenelements of integral operators", SIAM J. Numer. Anal. 23 (1986) 144-159.

[8] R. Alam, R. P Kulkarni and B. V. Limaye, "Boundedness of adjoint bases of approximate spectral subspaces and of associated block reduced resolvents", Numer. Funct. Anal. Optimiz. 17 (1996) 473-502.

[9] R. Alam, R. P Kulkarni and B. V. Limaye, "Accelerated spectral approximation", Math. Comp. 67 (1998) 1401-1422.

[10] R. Alam, R. P Kulkarni and B. V. Limaye, "Accelerated spectral refinement. Part I: Simple eigenvalue", J. Austral. Math. Soc. (Series B) 41 (2000) 487-507.

[11] D. Dellwo, "Accelerated spectral refinement with application to integral operators", SIAM J. Numer. Anal. 26 (1989) 1184-1193.

[12] B. V. Limaye, "Spectral perturbation and approximation with numerical experiments", in Proceeding of the Centre for Mathematical Analysis, Vol. 13, (Australian National University, 1986).

[13] M. T. Nair, "Computable error estimates for Newton's iterations for refining invariant subspaces", Indian J. Pure Appl. Math. 21 (1990) 1049-1054. 